\title{
Epithelioid sarcoma expresses epidermal growth factor receptor but gene amplification and kinase domain mutations are rare
}

\author{
Michael J Cascio ${ }^{1}$, Richard J O’Donnell ${ }^{2}$ and Andrew E Horvai ${ }^{1}$ \\ ${ }^{1}$ Department of Pathology, University of California, San Francisco, CA, USA and ${ }^{2}$ Department of Orthopaedic \\ Surgery, University of California, San Francisco, CA, USA
}

\begin{abstract}
Epithelioid sarcoma is a rare, malignant, soft tissue neoplasm that can be classified into proximal, distal and fibroma-like subtypes. Regardless of subtype, epithelioid sarcoma often shows morphologic and immunophenotypic evidence of epithelial differentiation. Current therapeutic strategies include surgical resection, amputation, radiation or chemotherapy, although the overall prognosis remains poor. The epidermal growth factor receptor (EGFR) is a novel therapeutic target in carcinomas. In some carcinomas, EGFR kinase domain mutations or gene amplification may correlate with response to specific inhibitors. EGFR expression has been reported in some sarcoma types, but expression, amplification and mutations have not been studied in epithelioid sarcoma. We evaluated 15 cases of epithelioid sarcoma from 14 patients for EGFR expression using immunohistochemistry, EGFR copy number aberration using fluorescence in situ hybridization and screened for mutations in the tyrosine kinase domain of the EGFR gene using direct sequencing. In all, 14 of the 15 epithelioid sarcomas (93\%) showed expression of EGFR by immunohistochemistry. A majority of the cases ( $n=11,73 \%)$ showed strong $(2+$ to $3+$ ) and homogeneous ( $>75 \%$ of cells) membrane staining. No amplification or polysomy of the EGFR gene or mutations of the tyrosine kinase domain of EGFR (exons 18-21) were detected. These results imply that although EGFR is expressed in most epithelioid sarcomas regardless of subtype, gene amplification and activating mutations in the tyrosine kinase domain appear to be rare or absent. Thus, the benefit of targeted therapy against EGFR in patients with epithelioid sarcoma remains to be determined.

Modern Pathology (2010) 23, 574-580; doi:10.1038/modpathol.2010.2; published online 29 January 2010
\end{abstract}

Keywords: epithelioid sarcoma; epidermal growth factor; immunohistochemistry

Epithelioid sarcoma is a rare, malignant soft tissue neoplasm that was initially described by Enzinger in 1970. ${ }^{1}$ Epithelioid sarcoma can be divided into three distinct morphologic subtypes: distal, proximal and fibroma-like. The distal, and most common, subtype typically arises in the distal extremities of young adults, with a predilection for the wrist, hand and fingers. The proximal subtype is generally found in the groin or trunk of older adults. Morphologically and immunophenotypically, these tumors mimic features of true epithelial neoplasms insofar as they stain for low- and high-molecular weight cytokeratins, as well as epithelial membrane antigen.

Correspondence: Dr AE Horvai, MD, PhD, Department of Pathology, University of California, San Francisco, 1600 Divisadero Drive B220, San Francisco, CA 94115-1656, USA.

E-mail: andho@itsa.ucsf.edu

Received 20 July 2009; revised 21 August 2009; accepted 24 August 2009; published online 29 January 2010
Ultrastructural analysis shows evidence of epithelial and myofibroblastic differentiation..$^{2-4}$ Recently, loss of expression of INI1, a core subunit of a chromatin remodeling complex involved in transcriptional regulation, has been documented in both distal and proximal-type epithelioid sarcomas, providing a possible mechanism of tumorigenesis. ${ }^{5,6}$ Current therapeutic strategies use wide surgical excision, radical resection, and in exceptional cases, amputation, followed by chemotherapy and/or radiation therapy. Regardless of subtype, the overall prognosis is poor, with local recurrence observed in up to $93 \%$ of patients, metastases in $30-45 \%$ and overall 10 -year survival of $42 \% .^{7}$

The human epidermal growth factor receptor (EGFR) (HER) family consists of four distinct type 1 receptor tyrosine kinases and includes HER1 (ErbB1, EGFR), HER2 (ErbB-2), HER3 (ErbB-3) and HER4 (ErbB-4). On ligand binding, these receptors form homo- or heterodimers with other HER members 
leading to autophosphorylation and activation of downstream signaling pathways involved in cell proliferation, apoptosis and angiogenesis. Consequently, aberrant EGFR signaling has been implicated in malignant transformation and tumor cell survival, especially in carcinomas, suggesting a therapeutic role for targeted EGFR inhibitors. Specifically, small molecule tyrosine kinase inhibitors and monoclonal antibodies against EGFR represent novel therapeutic approaches in colorectal adenocarcinoma, non-small cell lung carcinoma and squamous cell carcinoma of the head and neck. ${ }^{8-10}$ Previous studies have shown that mutations in the tyrosine kinase domain of EGFR (corresponding to exons 18-21) or amplification of the EGFR gene can be used as biomarkers for response to EGFR-targeted therapy or overall survival. ${ }^{11,12}$ Interestingly, recent evidence suggests that EGFR expression is not unique to carcinomas but may be present in malignant bone and soft tissue tumors, with a small subset showing aberrant EGFR gene copy number and/or mutations in the tyrosine kinase domain. ${ }^{13-18}$ However, the expression, amplification and mutation status of epithelioid sarcoma remains to be characterized.

To analyze a potential role for EGFR-targeted therapy, we evaluated 15 cases of epithelioid sarcoma from 14 patients, including examples of all three subtypes, for EGFR expression by immunohistochemistry, gene copy number aberration by fluorescence in situ hybridization (FISH), and mutations in the tyrosine kinase domain using PCR and direct sequencing. These data show that although EGFR expression is detectible by immunohistochemistry in the majority of epithelioid sarcomas, gene amplification and kinase domain mutations are rare to absent.

\section{Materials and methods}

\section{Case Selection}

The study was carried out according to guidelines set forth by the Institutional Review Board of the University of California, San Francisco and San Francisco General Hospital. We identified, retrospectively, 15 cases of epithelioid sarcoma from 14 patients in the pathology archives at our institution. The diagnosis was based on light microscopic, clinical and immunohistochemical features according to established criteria. $^{19,20}$ Routine immunohistochemistry for keratin, epithelial membrane antigen, CD34 and INI1 was evaluated in all cases. Clinical data were obtained from records in the Department of Orthopedic Surgery.

\section{Immunohistochemistry}

Immunohistochemistry was performed on 3-mmthick paraffin-embedded sections using EGFR
pharmDx (Dako, Carpinteria, CA, USA). All cases were stained and scored according to the manufacturer's recommendations. Briefly, when more than $1 \%$ of tumor cells showed partial or complete membranous staining of any intensity, the tumor was considered positive for EGFR. Staining intensity was then quantified from $1+$ to $3+$, with $>75 \%$ of cell staining defined as homogenous.

\section{Fluorescence In Situ Hybridization}

Dual-color FISH was performed on formalin-fixed paraffin-embedded, 5-mm-thick tissue sections using Vysis LSI EGFR SpectrumOrange and CEP 7 SpectrumGreen probes (Vysis, Abbott AG, Baar, Switzerland). Each sample was deparaffinized according to standard protocol, then $10 \mu \mathrm{l}$ of EGFR and centromere 7 ( $C E P 7)$ probes were applied to the tissue, cover slipped, and sealed with rubber cement. Slides were placed in Hybrite chamber (Vysis, Abbott AG), denatured for $6 \mathrm{~min}$ at $72{ }^{\circ} \mathrm{C}$ and hybridized overnight at $37^{\circ} \mathrm{C}$. The slides were scored by counting the EGFR and CEP7 signals in 50 cells and the average number of signals per cell was calculated. Polysomy and gene amplification were assessed according to previous published studies. ${ }^{15,21,22}$

\section{EGFR Mutation Analysis}

All specimens were assessed to confirm that the proportion of tumor in tissue sections reached $50 \%$. Necrotic areas were specifically avoided. Briefly, genomic tumor DNA was extracted from microdissected tumor tissue obtained from five to eight $10 \mu$ m-thick, FFPE sections using the QIAamp DNA Mini Kit (Qiagen, Valencia, CA, USA). Polymerase chain reaction was performed with $50 \mathrm{ng}$ template DNA, $0.2 \mathrm{mM}$ primer concentration, and Amplitaq Gold PCR Master Mix (Applied Biosystems, Foster City, CA, USA) for a total reaction volume of $20 \mu \mathrm{l}$. Forward and reverse primer sequences for EGFR exons 18, 19, 20 and 21 were as follows: EGFR18 S: $5^{\prime}$-GTGTCCTGGCACCCAAGC-3'; EGFR18 AS: 5'-TCCCCACCAGACCATGAGAG-3'; EGFR19 S: 5'-CCCAGTGTCCCTCACCTTCG-3'; EGFR19 AS: 5'-TGGAGATGAGCAGGGTCTAGAGCA-3'; EGFR20 S: 5'-GAAGGGGTCCATGTGCC-3'; EGFR20 AS: 5'TATCCCAGGAGCGCAGAC-3'; EGFR21 S: 5'-GAA CATGACCCTGAATTCGGAT- ${ }^{\prime}$ and EGFR21 AS: ${ }^{\prime}{ }^{\prime}$ CCTCCCCTGCATGTGTTAAAC-3'. PCR was carried out as follows: $95{ }^{\circ} \mathrm{C}$ for $10 \mathrm{~min}$, then 40 cycles consisting of $95^{\circ} \mathrm{C}$ for $1 \mathrm{~min}, 60^{\circ} \mathrm{C}$ for $1 \mathrm{~min}, 72^{\circ} \mathrm{C}$ for $1 \mathrm{~min}$ and a final extension step of $72^{\circ} \mathrm{C}$ for 5 min. PCR products were cleaned with ExoSAP-IT (USB, Cleveland, OH, USA) using the protocol provided by the manufacturer. Automated forward and reverse genomic DNA sequencing was performed using PCR primer sequences as above and according to published methods. ${ }^{16}$ Sequences 
obtained were compared with wild-type EGFR sequences obtained from NCBI using Sequencher software (Gene Codes Corporation, Ann Arbor, MI, USA).

\section{Results}

The clinical and pathologic characteristics of the tumors are summarized in Table 1 . The series consisted of 15 tumors from 14 patients (seven women and seven men) with a mean age of 42 years (range 20-76). The primary tumors were from: upper extremity $(n=5)$, lower extremity $(n=5)$, groin $(n=3)$ and chest $(n=1)$. One patient (patient 3$)$ had a lung metastasis, which was also included in our analysis. Histologic subtypes included distal $(n=7)$, proximal $(n=5)$ and fibroma-like $(n=2)$. By routine immunohistochemistry, all tumors $(n=15)$ expressed either keratin or epithelial membrane antigen and eight tumors expressed both. CD34 expression was identified in nine tumors and no tumors $(n=0)$ expressed INI1.

In all, 14 of the 15 (93\%) epithelioid sarcomas showed expression of EGFR by immunohistochemistry (Figure 1 and Table 1). In patient 3, both the primary and metastasis expressed EGFR. Eleven cases $(73 \%)$ showed strong $(2+$ to $3+)$ and homogenous ( $>75 \%$ of cells) membranous staining.

FISH was evaluated on 14 cases, including the primary and metastatic lesions of case 3 , and none showed amplification (EGFR:CEP7 ratio ranged from 0.93 to 1.08) or polysomy of the EGFR gene (all tumors contained two EGFR copies and two centromere seven signals, Figure 2 and Table 1).

In 12 tumors, the tyrosine kinase domain could be successfully sequenced. DNA of sufficient quantity or quality could not be obtained on the remaining cases. Mutation analysis of the tyrosine kinase domain (exons 18-21) of the EGFR gene was evaluated using PCR and direct sequencing. There were no point mutations, insertions or deletions detected in any case (Table 1).

\section{Discussion}

The mainstay of therapy for epithelioid sarcoma is surgical resection and, in some cases, adjuvant chemotherapy with alkylating and/or anthracycline agents and/or radiation therapy. ${ }^{23,24}$ In a recent review, positive prognostic indicators in epithelioid sarcomas included age younger than 16 years, local stage of disease, negative lymph node status and complete surgical resection. ${ }^{25}$ The high rate of local recurrence and distant metastasis observed in epithelioid sarcoma necessitates the development of improved adjuvant systemic therapies. Given the presence of morphologic and immunophenotypic features suggesting epithelial differentiation and lack of effective therapies currently available, therapeutic strategies targeting EGFR are promising. The present data indicate that although all three subtypes of epithelioid sarcoma express EGFR, kinase domain mutations and gene amplification are rare or absent. To our knowledge, this study is the first to report EGFR gene amplification and kinase mutation status in epithelioid sarcoma.

Recently, several types of bone and soft tissue tumors have been shown to express EGFR and, in some cases, also amplification of the EGFR gene or mutations in the tyrosine kinase domain. ${ }^{14-17}$ In two recent studies of bone and soft tissue neoplasms, EGFR expression was noted in approximately $20 \%$ of malignant tumors, with no expression documented in benign lesions, suggesting that EGFR is

Table 1 Clinicopathologic, immunohistochemical and genetic data of EGFR in epithelioid sarcoma

\begin{tabular}{|c|c|c|c|c|c|c|c|c|c|c|c|}
\hline \multirow[b]{2}{*}{ Pt. } & \multicolumn{5}{|c|}{ Clinical data } & \multirow[t]{2}{*}{$I H C$} & \multirow{2}{*}{$\begin{array}{c}\text { FISH } \\
\text { EGFR/CEP7 } \\
\text { ratio }\end{array}$} & \multicolumn{4}{|c|}{ Mutations } \\
\hline & Gender & Age & Location & $\begin{array}{l}\text { Size } \\
\text { (cm) }\end{array}$ & Туре & & & Exon 18 & Exon 19 & Exon 20 & Exon 21 \\
\hline 1 & $\mathrm{~F}$ & 43 & Hand & 3 & Distal & $2+$ & 0.99 & - & - & - & - \\
\hline 2 & $\mathrm{M}$ & 76 & Arm & 11 & Distal & $3+$ & 1.00 & - & - & - & - \\
\hline \multirow[t]{2}{*}{3} & $\mathrm{~F}$ & 20 & Thigh & 7 & Fibroma-like & $3+$ & 1.02 & ND & ND & ND & ND \\
\hline & & & Lung & & & $2+$ & 0.95 & - & - & - & - \\
\hline 4 & $\mathrm{~F}$ & 52 & Forearm & 4 & Distal & $3+$ & 0.98 & - & - & - & - \\
\hline 5 & $\mathrm{~F}$ & 20 & Knee & 2 & Distal & 0 & 1.03 & - & - & - & - \\
\hline 6 & $\mathrm{~F}$ & 45 & Groin & 3 & Proximal & $3+$ & 0.93 & - & - & - & - \\
\hline 7 & $\mathrm{M}$ & 21 & Foot & 3 & Distal & $3+$ & 0.96 & - & - & - & - \\
\hline 8 & $\mathrm{M}$ & 50 & Knee & 11 & Proximal & $3+$ & 1.02 & - & - & - & - \\
\hline 9 & $\mathrm{M}$ & 61 & Chest wall & 8 & Proximal & $2+$ & 0.96 & - & - & - & - \\
\hline 10 & $\mathrm{~F}$ & 44 & Groin & 7 & Proximal & $3+$ & 1.00 & - & - & - & - \\
\hline 11 & $\mathrm{~F}$ & 22 & Elbow & 4 & Distal & $3+$ & 1.02 & - & - & - & - \\
\hline 12 & $\mathrm{M}$ & 44 & Groin & 5 & Proximal & $1+$ & 1.08 & - & - & - & - \\
\hline 13 & $\mathrm{M}$ & 42 & Thigh & 7 & Fibroma-like & $2+$ focal & 1.06 & ND & ND & ND & ND \\
\hline 14 & $\mathrm{M}$ & 49 & Shoulder & 3 & Distal & $1+$ focal & ND & ND & ND & ND & ND \\
\hline
\end{tabular}

CEP7: centromere 7; EGFR, epidermal growth factor receptor gene; F, female; FISH, fluorescence in situ hybridization; IHC, immunohistochemistry; M, male; ND, not done; Pt., patient; —, no mutation detected. 

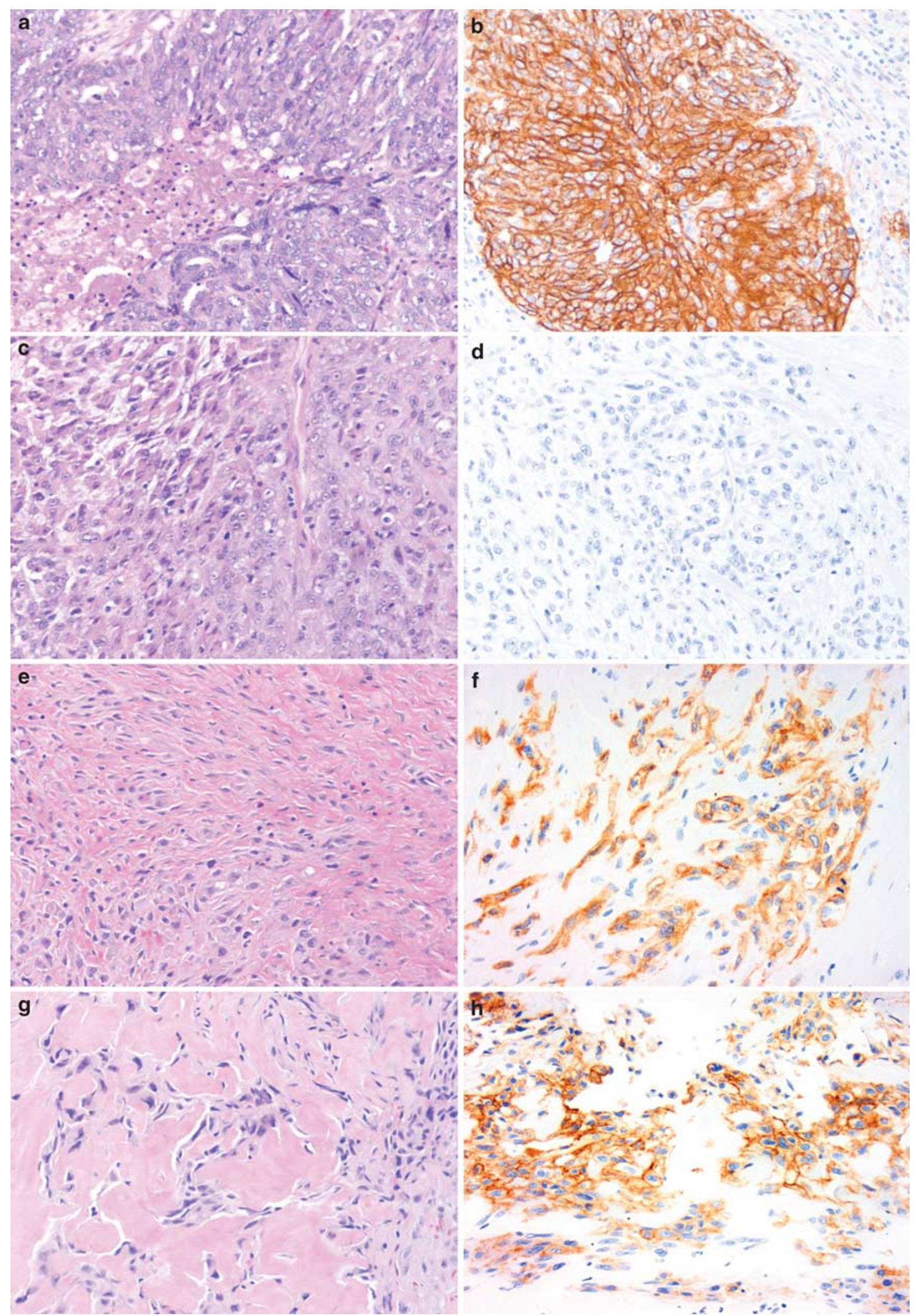

Figure 1 Hematoxylin and eosin (H\&E) sections and corresponding EGFR immunohistochemistry (original magnification $\times 400$ ). (a, b) Case 10, proximal-type with 3 + EGFR staining; (c, d) case 5, distal-type with no EGFR staining; (e, f) case 4, distal-type with 3 + EGFR staining; and $(\mathbf{g}, \mathbf{h})$ case 13, fibroma-like with $2+$ focal EGFR staining. 

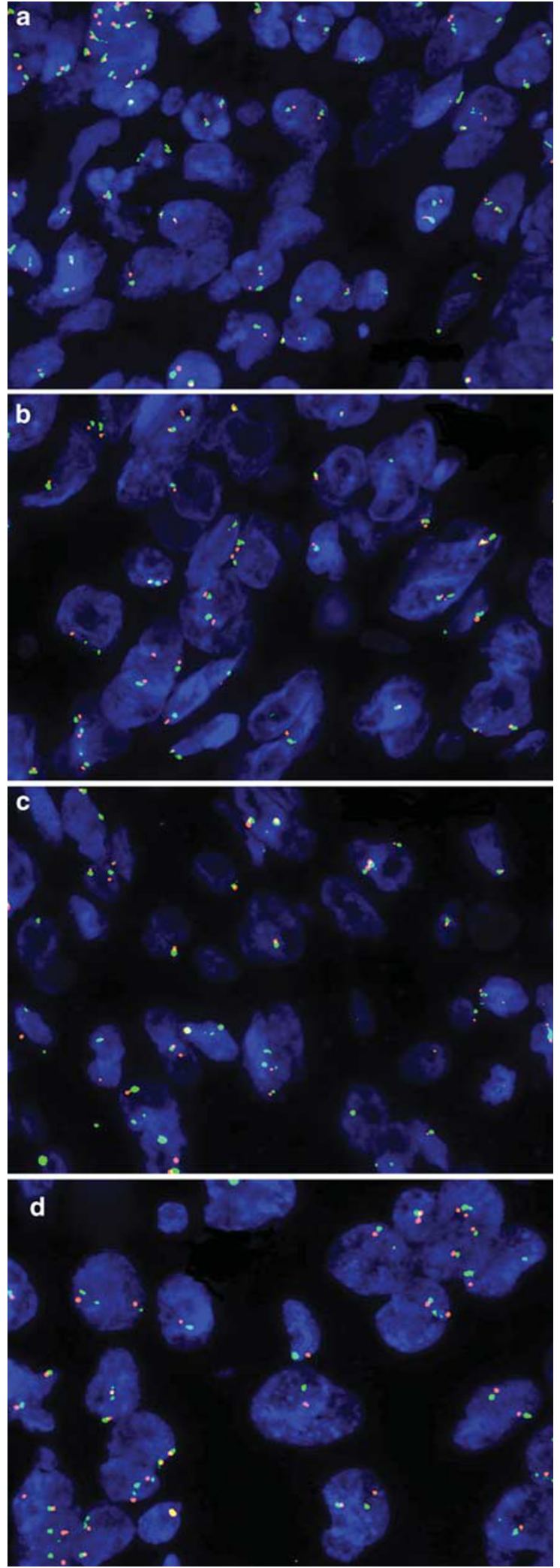

Figure 2 FISH for EGFR. The presence of approximately equal copy numbers of the EGFR gene (orange signal) relative to the centromere of chromosome 7 (CEP7, green signal) implies no amplification of the EGFR gene; (a) case 10, (b) case 5, (c) case 9 and $(\mathbf{d})$ case 8 . (Original magnification $\times 1000$ ). preferentially expressed in sarcomas. ${ }^{14,15}$ However, in general, kinase domain mutations in sarcomas are rare. ${ }^{26,27}$ Synovial sarcoma, like epithelioid sarcoma, shows morphologic and immunophenotypic features of epithelial differentiation. It is perhaps not surprising, therefore, that EGFR is often expressed in synovial sarcoma. ${ }^{18}$ However, in a series of 13 synovial sarcomas, Bode et $a 1^{16}$ found normal EGFR gene copy numbers in all cases and missense mutations in the kinase domain in only two cases. In the present series, epithelioid sarcomas showed expression of EGFR by immunohistochemistry, including strong, homogenous staining in the majority of cases, but absence of either gene amplification or kinase domain mutations. Taken together, the above data suggest that (1) EGFR amplification and kinase domain mutations in sarcomas are rare even in those tumors with epithelial morphology or immunophenotype and (2) immunohistochemical detection of EGFR expression in these sarcomas does not reliably predict gene amplification or kinase domain mutations.

Many previous studies have shown an inconsistent correlation between EGFR protein expression and response to tyrosine kinase inhibitor therapy in other tumor types. ${ }^{28}$ In theory, at least, targeted therapy is most effective in tumors that not only express EGFR but are dependent on EGFR signaling for growth and survival. Increased EGFR activation can arise through mutational events or gene amplification, however, constitutive activation, as observed with KIT mutations in gastrointestinal stromal tumor, is not a defining feature of mutations in the tyrosine kinase domain of EGFR. Lynch et $a l^{29}$ initially have shown that mutant EGFR has enhanced epidermal growth factor-dependent activation and increased sensitivity to gefitinib in vitro. Phase II clinical trails have revealed that deletion of exon 19 and L858R mutations are major markers of response to treatment in advanced non small cell lung cancer. ${ }^{30-32}$ In the present series of epithelioid sarcomas, no mutations or deletions were detected. These findings suggest that, based on current paradigms of assessing responsiveness to EGFRtargeted therapies in carcinomas, it is uncertain whether epithelioid sarcomas would show a meaningful response to EGFR tyrosine kinase inhibitors. Nevertheless, one study in refractory, advanced nonsmall cell lung carcinoma has shown EGFR protein expression as a biomarker predictive of better survival, ${ }^{33}$ suggesting that EGFR expression may have at least some clinical implication in sarcomas.

Further complicating the use of EGFR status as a predictive biomarker are data from several studies in metastatic colorectal carcinoma, which show that activating mutations in KRAS are predictive of unresponsiveness to therapies with cetuximab and pantimumab, regardless of EGFR mutation or gene amplification status. ${ }^{34}$ Recently, an in vitro study found that erlotinib-sensitive cell lines had increased expression of E-cadherin, with drug-resistant cell 
lines possessing high levels of delta-crystallin enhancer binding factor 1 (an E-cadherin repressor) and low levels of E-cadherin expression, suggesting that these may be potentially useful biomarkers. ${ }^{35}$ Stat-3 activation was associated with sarcomas expressing EGFR and/or having abnormal EGFR gene copy numbers by FISH, providing a potential target for the development of novel therapies. ${ }^{14}$ Thus, evaluation of additional biomarkers in malignant bone and soft tissue tumors, including epithelioid sarcoma, will be necessary to determine appropriate patient selection for the use of EGFR-targeted therapies.

Currently, phase II clinical trials are underway to evaluate EGFR monoclonal antibody or tyrosine kinase inhibitors in the treatment metastatic or locally advanced bone and soft tissue sarcomas (http://clinicaltrials.gov). In our series, we have shown that epithelioid sarcomas express EGFR by immunohistochemistry, but do not harbor tyrosine kinase domain mutations or increased EGFR gene copy number. Additional studies are needed to determine whether patients will benefit from the use of EGFR-targeted therapies and to further elucidate biomarkers predictive of response to therapy or clinical outcome.

\section{Acknowledgement}

We thank the staff of the UCSF Immunopathology Laboratory and Molecular Diagnostics Laboratory for their technical assistance.

\section{Disclosure/conflict of interest}

The authors declare no conflict of interest.

\section{References}

1 Enzinger FM. Epithelioid sarcoma. A sarcoma simulating a granuloma or a carcinoma. Cancer 1970;26: 1029-1041.

2 Eyden B, Wang G, Yao L. Epithelioid sarcoma: a case report with ultrastructural confirmation of myofibroblastic differentiation based on fibronexus junctions. Ultrastruct Pathol 2009;33:61-66.

3 Fisher C. Epithelioid sarcoma: the spectrum of ultrastructural differentiation in seven immunohistochemically defined cases. Hum Pathol 1988;19:265-275.

4 Persson S, Kindblom LG, Angervall L. Epithelioid sarcoma. An electron-microscopic and immunohistochemical study. Appl Pathol 1988;6:1-16.

5 Hornick JL, Dal Cin P, Fletcher CD. Loss of INI1 expression is characteristic of both conventional and proximal-type epithelioid sarcoma. Am J Surg Pathol 2009;33:542-550.

6 Orrock JM, Abbott JJ, Gibson LE, et al. INI1 and GLUT-1 expression in epithelioid sarcoma and its cutaneous neoplastic and nonneoplastic mimics. Am J Dermatopathol 2009;31:152-156.
7 Fisher C. Epithelioid sarcoma of Enzinger. Adv Anat Pathol 2006;13:114-121.

8 Han SW, Kim TY, Hwang PG, et al. Predictive and prognostic impact of epidermal growth factor receptor mutation in non-small-cell lung cancer patients treated with gefitinib. J Clin Oncol 2005;23:2493-2501.

9 Loong HH, Ma BB, Chan AT. Update in antiepidermal growth factor receptor therapy in the management of metastatic colorectal cancer. J Oncol 2009;2009: 967920.

10 Mehra R, Cohen RB, Harari PM. EGFR inhibitors for the treatment of squamous cell carcinoma of the head and neck. Curr Oncol Rep 2008;10:176-184.

11 Cappuzzo F, Ligorio C, Janne PA, et al. Prospective study of gefitinib in epidermal growth factor receptor fluorescence in situ hybridization-positive/phosphoAkt-positive or never smoker patients with advanced non-small-cell lung cancer: the ONCOBELL trial. J Clin Oncol 2007;25:2248-2255.

12 Chung CH, Ely K, McGavran L, et al. Increased epidermal growth factor receptor gene copy number is associated with poor prognosis in head and neck squamous cell carcinomas. J Clin Oncol 2006;24: $4170-4176$.

13 Gusterson B, Cowley G, McIlhinney J, et al. Evidence for increased epidermal growth factor receptors in human sarcomas. Int J Cancer 1985;36:689-693.

14 Dobashi Y, Suzuki S, Sugawara H, et al. Involvement of epidermal growth factor receptor and downstream molecules in bone and soft tissue tumors. Hum Pathol 2007;38:914-925.

15 Dobashi Y, Takei N, Suzuki S, et al. Aberration of epidermal growth factor receptor expression in bone and soft-tissue tumors: protein overexpression, gene amplification and activation of downstream molecules. Mod Pathol 2004:17:1497-1505.

16 Bode B, Frigerio S, Behnke S, et al. Mutations in the tyrosine kinase domain of the EGFR gene are rare in synovial sarcoma. Mod Pathol 2006;19:541-547.

17 Sato O, Wada T, Kawai A, et al. Expression of epidermal growth factor receptor, ERBB2 and KIT in adult soft tissue sarcomas: a clinicopathologic study of 281 cases. Cancer 2005;103:1881-1890.

18 Tawbi H, Thomas D, Lucas DR, et al. Epidermal growth factor receptor expression and mutational analysis in synovial sarcomas and malignant peripheral nerve sheath tumors. Oncologist 2008;13:459-466.

19 Fletcher CDM, Unni KK, Mertens F, (eds). Pathology and Genetics of Tumours of Soft Tissue and Bone. IARC Press: Lyon, France, 2002, pp 173-174.

20 Weiss SW, Goldblum JR. Enzinger and Weiss's Soft Tissue Tumors, 5th edn. Mosby: St Louis, 2008, pp 1191-1203.

21 Buckley AF, Burgart LJ, Kakar S. Epidermal growth factor receptor expression and gene copy number in fibrolamellar hepatocellular carcinoma. Hum Pathol 2006;37:410-414.

22 Buckley AF, Burgart LJ, Sahai V, et al. Epidermal growth factor receptor expression and gene copy number in conventional hepatocellular carcinoma. Am J Clin Pathol 2008;129:245-251.

23 Spillane AJ, Thomas JM, Fisher C. Epithelioid sarcoma: the clinicopathological complexities of this rare soft tissue sarcoma. Ann Surg Oncol 2000;7: 218-225.

24 Baratti D, Pennacchioli E, Casali PG, et al. Epithelioid sarcoma: prognostic factors and survival in a series of 
patients treated at a single institution. Ann Surg Oncol 2007;14:3542-3551.

25 Jawad MU, Extein J, Min ES, et al. Prognostic factors for survival in patients with epithelioid sarcoma: 441 cases from the SEER database. Clin Orthop Relat Res 2009;467:2939-2948.

26 Sihto H, Puputti M, Pulli L, et al. Epidermal growth factor receptor domain II, IV, and kinase domain mutations in human solid tumors. J Mol Med 2005;83:976-983.

27 Baird K, Davis S, Antonescu CR, et al. Gene expression profiling of human sarcomas: insights into sarcoma biology. Cancer Res 2005;65:9226-9235.

28 Le Tourneau C, Vidal L, Siu LL. Progress and challenges in the identification of biomarkers for EGFR and VEGFR targeting anticancer agents. Drug Resist Updat 2008;11:99-109.

29 Lynch TJ, Bell DW, Sordella R, et al. Activating mutations in the epidermal growth factor receptor underlying responsiveness of non-small-cell lung cancer to gefitinib. N Engl J Med 2004;350:2129-2139.

30 Inoue A, Suzuki T, Fukuhara T, et al. Prospective phase II study of gefitinib for chemotherapy-naive patients with advanced non-small-cell lung cancer with epidermal growth factor receptor gene mutations. J Clin Oncol 2006;24:3340-3346.
31 Mitsudomi T, Kosaka T, Endoh H, et al. Mutations of the epidermal growth factor receptor gene predict prolonged survival after gefitinib treatment in patients with non-small-cell lung cancer with postoperative recurrence. J Clin Oncol 2005;23:2513-2520.

32 van Zandwijk N, Mathy A, Boerrigter L, et al. EGFR and KRAS mutations as criteria for treatment with tyrosine kinase inhibitors: retro- and prospective observations in non-small-cell lung cancer. Ann Oncol 2007;18:99-103.

33 Hirsch FR, Varella-Garcia M, Bunn Jr PA, et al. Molecular predictors of outcome with gefitinib in a phase III placebo-controlled study in advanced nonsmall-cell lung cancer. J Clin Oncol 2006;24: 5034-5042.

34 Jimeno A, Messersmith WA, Hirsch FR, et al. KRAS mutations and sensitivity to epidermal growth factor receptor inhibitors in colorectal cancer: practical application of patient selection. J Clin Oncol 2009;27:1130-1136.

35 Haddad Y, Choi W, McConkey DJ. Delta-crystallin enhancer binding factor 1 controls the epithelial to mesenchymal transition phenotype and resistance to the epidermal growth factor receptor inhibitor erlotinib in human head and neck squamous cell carcinoma lines. Clin Cancer Res 2009;15:532-542. 